\title{
Revisiting Sports Dentistry with a Critical Appraisal
}

\author{
Urmi Ghone ${ }^{1}$, Gargi Sarode ${ }^{2}$, Sachin Sarode ${ }^{3}$, Shankargauda Patil ${ }^{4}$
}

\begin{abstract}
Dental traumas caused due to sports and other athletic activities are the connecting bridge between sports and dentistry. Studies show that $13-39 \%$ of all dental injuries are accounted for sports, out of which 11-18\% are maxillofacial injuries. This editorial broadly discusses the interconnection between sports and dentistry. It highlights the importance of Parents', children's, sportspersons', and even dental practitioners' education on this topic of sports dentistry. The significant need for "team dentist" in schools and professional sports teams is also brought into the spotlight.

Keywords: Dentistry, Injury, Sport, Trauma.

The Journal of Contemporary Dental Practice (2021): 10.5005/jp-journals-10024-3007
\end{abstract}

India is becoming more health and fitness conscious, and thus, more and more people are becoming involved in sports and related activities, from playing national and international games to occasional sports activities. With the increase in these sports activities, there is an increase in the cases of orofacial trauma. Thus, dentists must be aware of the sports-related orofacial traumas and should be capable to give first aid and respond to the specialized needs involved in the sports injuries and the standard care that is needed. Moreover, knowledge acquisition will be of paramount importance for disseminating appropriate precautionary measures to the people involved in sports. Specialists in oral and maxillofacial surgery, endodontics, orthodontics, esthetic dentistry, prosthodontics, and operative dentistry can actively get involved in this field.

Sports dentistry is the most recent and forthcoming field in dentistry. It largely deals with the management and the prevention of athletic or sports-related orofacial injuries and associated oral disorders. The sports dentist assists an athlete or a sportsperson in the prevention, diagnosis, and treatment of these orofacial injuries. Most of the sportspersons like soccer players and gymnasts approach sporting goods retailers for their preventive dental requirements. This is due to the minimal awareness of sports dentistry among the athletes and other sportspersons.

Sports teams and athletes in some countries still fall short of access to special and structural dental care under the eyes of professionals. In many foreign countries like Canada and parts of Europe, dentists today are progressively being considered as essential members of sports medical team. Many dentists abroad have amalgamated their passion-sports-with their careerdentistry. Moreover, sports dentistry in other countries is taken up as a postgraduate course.

Orofacial trauma in sports and athletics is the major bridge between sports and dentistry. It has two major components: (1) treatment of orofacial injuries and (2) prevention of sports-related orofacial injuries.

Due to mandatory regulation, sports activities start early at the school level. During the regular sports played in the school, it is very common for the kids to get orofacial traumas, oromaxillofacial region being the most disposed and common area of injury. Dentists tend to deal with these types of patients having different types of fractures of tooth and orofacial bones. Hence, to provide
${ }^{1-3}$ Department of Oral Pathology and Microbiology, Dr. DY Patil Dental College and Hospital, Dr. DY Patil Vidyapeeth, Pimpri, Pune, Maharashtra, India

${ }^{4}$ Department of Maxillofacial Surgery and Diagnostic Sciences, Division of Oral Pathology, College of Dentistry, Jazan University, Jazan, Saudi Arabia

Corresponding Author: Gargi Sarode, Department of Oral Pathology and Microbiology, Dr. DY Patil Dental College and Hospital, Dr. DY Patil Vidyapeeth, Pimpri, Pune, Maharashtra, India, e-mail: gargi14@gmail. com

How to cite this article: Ghone U, Sarode G, Sarode S, et al. Revisiting Sports Dentistry with a Critical Appraisal. J Contemp Dent Pract 2021;22(2):105-106.

Source of support: Nil

Conflict of interest: None

comprehensive care for a sports injury, a dentist must have the basic knowledge of providing the preliminary care and treatment (oral surgery, endodontics, orthodontics, prosthodontics, operative dentistry, and patient behavioral management) to the patients and/ or proper referral. ${ }^{1}$

Fracture to the bones, tooth-related injuries like fractures, intrusion, luxation, crown and/or root fractures, temporomandibular joint injuries, tooth wear, abrasions, and lacerations are some of the injuries that may occur during sports. These injuries during the sports should be prevented. Necessary preventive measures like use of mouth guards, helmets, and other protective gears are believed to reduce the injuries faced in sports. Looking at all the aforementioned aspects, it is envisaged that sports in-charge at schools should be made aware of the modes of possible dental sports injuries and their preventions using appropriate aids. Hence, a brief training and sensitization sessions at regular intervals should be conducted for the sports teachers and physical trainers in the schools.

Studies show that $13-39 \%$ of all dental injuries are sports related, out of which $11-18 \%$ approximately are sports-related maxillofacial injuries, which might be due to direct hit or playerto-player contact. ${ }^{2,3}$ Some studies have reported that maxillary central incisors are the most commonly affected teeth due to sports injury. Certain risk factors can be accountable for 
the unpleasant events encountered during sports and can be broadly classified as extrinsic and intrinsic factors. ${ }^{3,4}$ Extrinsic factors include training mistakes, incorrect methods of preparing for competitions, climatic conditions, and quality of playing surfaces. Intrinsic factors include the participant-related factors like age, gender, comorbidities like cerebral palsy and epilepsy, and participants with increased body mass and nutritional status. $^{3}$

Therefore, it is important for the dental professionals to interact with the sports world, which might include the opportunities for dentists in the field of sports. Jackson proposed the great need for "team dentists" from high school to professional sports teams. ${ }^{5}$ Although these are isolated events in the literature, comprehensive evidence-based information in the field of sports dentistry is the need of the hour. A systematic review or meta-analysis is mandated to further bring more clarity on this aspect including the type and nature of orofacial injuries.

On interviewing a sports education company (which provides a structured platform to deliver physical education (PE) lessons to children, parents, teachers, and schools) about the possible sports-related orofacial trauma and sports dentistry, they seemed quite unaware of the existence of this field in dentistry. This and the increasing sports-related dental traumas tell us that India lacks behind in this field due to very less awareness among individuals. Hence, proper training of teachers and emergency management kits are equally important.

The athletes should be made aware of this field of dentistry and shall be encouraged to have dental assessments or screenings during the off-seasons so as to minimize the risk of any flare-ups during competitions. Dental professionals shall also spread the necessary awareness among their fellow dentists and patients. Lack of awareness is the key reason why our country is unable to bring sports dentistry into practice.

With the rising anxiety about the occurrences of sports-related dental injuries in children, adolescents, and adults, certain interest must be taken by the dentists in educating people on the use of protective equipment during sports activity to prevent injuries related to sports. Dentists should actively get involved in designing, fabricating, and manufacturing protective gears to avoid orofacial injuries. Properly fitted customized mouth guards during sports should be made mandatory, and people should be educated about the same. Prosthodontists and orthodontists play a crucial role in this. Oromaxillofacial gears capable of aiding the sustained release of glucose or certain drugs like analgesics could also be designed to avoid dehydration and other conditions.

Prevention of sports-related traumatic orofacial injuries should be done, and awareness should be made among the other medical professionals and among the sportspersons by the dentists. Currently, helmets, mouth guards, and face masks are made compulsory in some sports to reduce the occurrence and severity of sports-related traumatic injuries of head, face, and mouth of a sportsperson. Mouth protection is believed to be advantageous and most required in sports where there is high risk of injuries like in football and boxing.

Specific counseling regarding the prevention of sports-related trauma, malocclusion, and use of mouth protectors should be done to the athletes by their sports dentists. It is the duty of the dentists to spread awareness in the population, teachers and students in school, and children who indulge in regular sports activities. Dentists should also participate in educational and public service activities and help in spreading awareness in the society. Awareness among dental fraternity is also necessary as many dentists are not aware of their role in the field of sports. Importance of sports dentistry should also be included in the curriculum of the dental undergraduate education. There can be a provision of certificate or diploma courses in the field of sports dentistry.

Thus, sports dentistry comprises preventive and treatment aspects of orofacial sports injuries and their related oral disorders. The pediatric dentist should also impart knowledge regarding sports-related injuries in children and the necessary preventive measures needed for it. Recently, on the World Mental Health Day, Bengaluru Football Club became the first Indian club to add mental health experts to their team. ${ }^{6}$ In the same way, on World Dentist's Day (February 9), dentists can spread the awareness among the athletes and sportspersons so that a dentist's team can also be appointed to take the utmost responsibility to identify, educate, and provide the necessary preventive measures to the sportspersons.

\section{References}

1. Andresean JO, Andresean FM. Textbook of Color Atlas of Traumatic Injuries to the Teeth, 3rd ed. Copenhagen: Munksgaard; 1994.

2. Sane J. Maxillofacial and dental injuries in contact team sports. Proc Finn Dent Soc 1988;84(Suppl 6-7):1-45.

3. Ramagoni NK, Singamaneni VK, Rao SR, et al. Sports dentistry: a review. J Int Soc Prev Community Dent 2014;4:S139-S146. DOI: 10.4103/2231-0762.149019.

4. Taimela S, Kujala UM, Osterman K. Intrinsic risk factors and athletic injuries. Sports Med 1990;9(4):205-215. DOI: 10.2165/00007256199009040-00002.

5. Winters JE. Sports dentistry: the profession's role in athletics. JADA 1996;127(6):810-811. DOI: 10.14219/jada.archive.1996.0322.

6. https://www.bengalurufc.com/2020/club-news/bengaluru-fclaunch-mental-health-care-programme-team/ (accessed on 13th November 2020). 